\title{
Treatment of EGFR positive lung adenocarcinoma in a heart transplanted patient
}

\author{
Ana Hecimovic1, Andrea Vukic Dugac ${ }^{1,2}$, Mateja Jankovic Makek ${ }^{1,2}$, Maja Cikes ${ }^{2,3}$, Miroslav Samarzija ${ }^{1,2}$, \\ Marko Jakopovic ${ }^{1,2}$ \\ ${ }^{1}$ Department for Lung Diseases, University Hospital Centre Zagreb; ${ }^{2}$ School of Medicine, University of Zagreb; \\ ${ }^{3}$ Department of Cardiology, University Hospital Centre Zagreb, Croatia
}

\begin{abstract}
Lung cancer incidence in heart transplant patients is higher than in general population and correlates with smoking history. EGFR-mutations are more frequent in adenocarcinoma and among non-smoking women but incidence in solid organ transplanted patients is still not known. We present case of a 65 -yearold ex-smoker male with history of heart transplantation and EGFR positive metastatic lung adenocarcinoma. At admission he was in a severe clinical condition and treatment with erlotinib was
\end{abstract}

Correspondence: Ana Hecimovic, Department for Lung Diseases, University Hospital Centre, Jordanovac 104, 10000 Zagreb, Croatia. Tel. +385.1.2385231 - Fax: +385.1.2385388

E-mail: anahecimovic1978@gmail.com

Keywords: Lung cancer; EGFR mutation; heart transplantation; tyrosine kinase inhibitor; erlotinib

Contributions: AH, MJ, MS, conception and design of the study, manuscript drafting, critical revision; MJM, AVD, MC, data collection, critical revision of the article. All the authors read and approved the final version of the article.

Conflict of Interest: A. Hecimovic reported personal fees from Roche, Boehringer Ingelheim and MSD outside the submitted work. M Jakopovic and M. Samarzija reported personal fees from Roche, Boehringer Ingelheim, Novartis, Astra Zeneca and Bristol-Mayers, outside the submitted work. A.Vukic Dugac reported personal fees from Astra Zeneca, Novartis, Boehringer Ingelheim and Teva, outside the submitted work. M. Cikes declare no conflict of interest.

Funding: This research did not receive any specific grant from funding agencies in the public, commercial or not-of-profit sectors.

Received for publication: 1 January 2019.

Accepted for publication: 12 May 2019.

CCopyright A. Hecimovic et al., 2019

Licensee PAGEPress, Italy

Monaldi Archives for Chest Disease 2019; 89:1023

doi: 10.4081/monaldi.2019.1023

This article is distributed under the terms of the Creative Commons Attribution Noncommercial License (by-nc 4.0) which permits any noncommercial use, distribution, and reproduction in any medium, provided the original author(s) and source are credited. started. Initially he had good clinical and radiologic response to treatment with only grade 1 side effects. Data about drug interactions between cyclosporine and erlotinib are insufficient but we have to take this interaction into consideration during treatment because both drugs are substrates and inhibitors of CYP34A. In our case erlotinib was safe and well tolerated drug, there were no relevant toxicity, but close monitoring and dose reduction of cyclosporine was needed.

\section{Introduction}

Malignancies are one of the complications in transplant patients and are now leading cause of mortality in this population [1]. Among heart transplanted (HT) recipients incidence of lung cancer is higher than in general population and correlates with smoking history [2-5]. Frequency of epidermal growth factor receptor (EGFR)-mutation is higher in adenocarcinoma, nonsmoking women and East Asian ethnicity [6]. There is no published data in literature about frequency of EGFR positive patients among solid organ transplanted patients.

Treatment options for lung cancer among transplanted patients are the same as in the general population. Patients with EGFR positive lung carcinoma are treated with tyrosine kinase inhibitors (TKI). Erlotinib is one of the TKI drugs and is mostly metabolized by cytochrome P450 CYP3A4 and CYP1A1 [7]. Transplant patients with lung cancer have shown poorer overall survival compared to non-transplanted patients according to the limited data [8].

We report a case of HT patient with stage IV EGFR positive lung adenocarcinoma treated with erlotinib. Erlotinib was well tolerated in combination with immunosuppressive therapy with no clinically significant toxicity.

\section{Case Report}

A 65-year-old man, ex-smoker (40 pack/years), was admitted to our Department after metastatic lung cancer was diagnosed during his stay in Department of Cardiology. In April 2012 he had heart transplantation due to ischemic cardiomyopathy and was in a stable condition without signs of transplant rejection. He was treated with cyclosporine, micophenolat-mophetyl and a low dose of prednisolone as immunosuppressive therapy. His first symptom was exertional dyspnea followed by dry cough, low-grade fever, and he lost 15 kilograms till admission to Department. 
At admission to our Department he was in a sever condition, tachypneic (respiratory rate $30 / \mathrm{min}$ ) and severely hypoxemic with $\mathrm{PaO}_{2} 33 \mathrm{mmHg}$. Laboratory test showed elevated urea level $18,9 \mathrm{mmol} / \mathrm{L}$, creatinine level $132 \mu \mathrm{mol} / \mathrm{L}, \mathrm{LDH} 884 \mathrm{U} / \mathrm{L}, \mathrm{C}$ reactive protein level $72.3 \mathrm{mg} / \mathrm{L}$ and other findings were within normal limits.

His chest X-ray revealed bilateral interstitial lung changes at the time of fist symptoms started. Heart transplant rejection and infection were excluded by heart biopsy and there was no improvement on antibiotic and antiviral treatment. Since his condition get worse chest computed tomography (CT) scan was done and it showed multiple micronodules 1 to $3 \mathrm{~mm}$ in diameter, thickening of interlobular and intralobular septa and a few areas of ground glass and bilateral small pleural effusions (Figure 1). Bronchoscopy with bronchoalveolar lavage and transbronchial biopsy was performed and pathology finding was lung adenocarcinoma. Molecular analysis for EGFR mutations showed mutation L858R on exon 21. According to the diagnostic procedures definitive diagnosis was stage IV lung adenocarcinoma with lymphangiosis carcinomatosa of bo th lungs and bilateral pleural carcinosis.

Despite his quite severe condition we decided to start treatment with tyrosine kinase inhibitor (TKI) erlotinib since he had EGFR L585R positive mutation. His condition improved after only a week and he was discharged from the hospital.

One month after starting the treatment with erlotinib there was no need for changing the dose of cyclosporine. He was receiving $70 \mathrm{mg}$ cyclosporine twice daily and his cyclosporine blood level was between 80 and $90 \mu \mathrm{g} / \mathrm{L}$. After that his cyclosporine blood level started to rise above targeted level of $100 \mu \mathrm{g} / \mathrm{L}$ and the highest blood level of cyclosporine was above $220 \mu \mathrm{g} / \mathrm{L}$. Cyclosporine dose was gradually reduced and three months after starting erlotinib the dose of cyclosporine was 40 $\mathrm{mg}$ in the morning and $30 \mathrm{mg}$ in the evening.

Chest CT revealed significant reduction of interstitial and micronodular lung changes (Figure 2) with clinical improvement and oxygen level on room air of $74 \mathrm{mmHg}$ two months after beginning of the treatment. He had only grade 1 skin side effects and diarrhea for only two days during the first month of treatment without erlotinib side effects during further treatment.

Unfortunately four months after beginning of treatment, progression of left sided pleural effusion and interstitial opacities was revealed on chest X-ray and his condition was severely deteriorated. Erlotinib treatment was stopped and he died about four and a half months after establishing the diagnosis and starting the treatment.

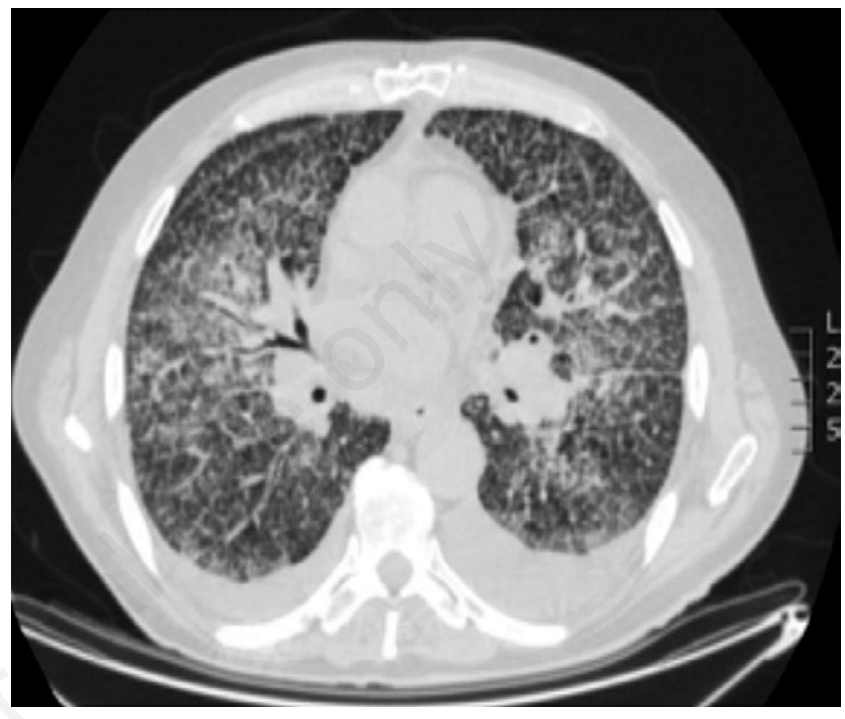

Figure 1. Chest CT scan before definitive diagnosis was established: multiple micronodules 1 to $3 \mathrm{~mm}$ in diameter, thickening of interlobular and intralobular septa with few areas of ground glass and minimal bilateral pleural effusions.
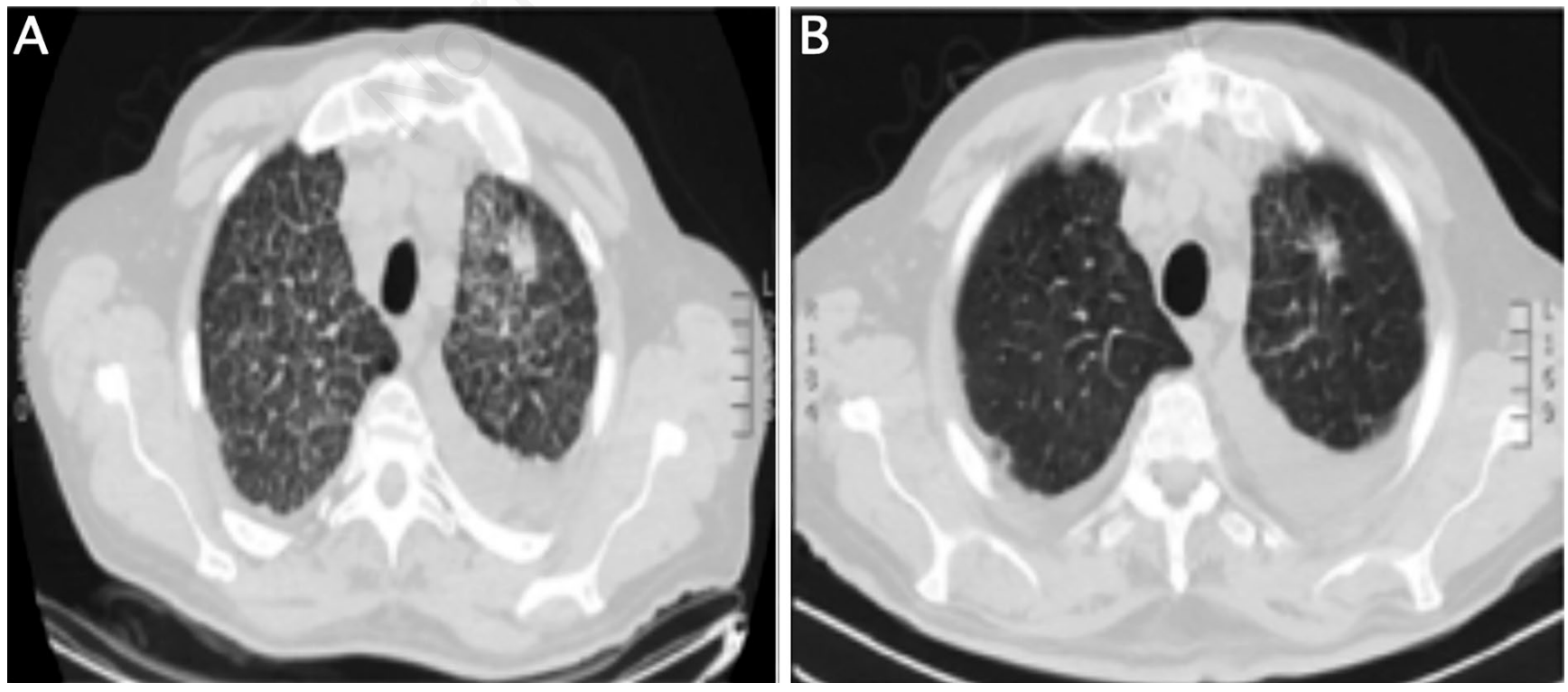

Figure 2. Comparison of CT scan before treatment (A) and two months after treatment (B). 


\section{Discussion}

Nowadays we have longer allograft survival due to advances in immunosuppressive regiments which leads to collateral emergence of long-term post-transplant complications and malignancies are one of the most frequent.

Lung cancer is one of the most frequent malignancies in heart transplanted patients [4]. In heart transplant recipients', higher incidence of lung cancer is not necessarily a consequence of the immunosuppression therapy because we can approach to these patients as a selected group of patients who have another risk factor like cigarette smoking. In published data cigarette smoking is linked to lung cancer development in heart transplant recipients which can be explained by augmentation of ischemic heart failure caused by tobacco consumption as a background factor of the heart transplantation [3].

Most of the published studies showed that lung cancer in organ transplant recipients is presented in late stage of disease [1,4]. Our patient correlate with that because at admission he had stage IV of lung cancer although he had frequent monitoring after transplantation. Treatment approach in heart transplanted patient is the same as in general population and it depends on histologic type of the tumor, mutation status, stage of the disease and patient's performance status. In our case, according to mutation status where we had EGFR L585R positive mutation, treatment with TKI was indicated. We decided to start treatment with erlotinib in spite of poor performance status and severe respiratory insufficiency. One of the things that differs this group of patients from general population is immunosuppressive therapy that they are taking. In our case cyclosporine and micophenolat-mophetyl were two drugs that we have to consider for possible drug interactions with erlotinib. Cyclosporine is, like erlotinib, CYP3A4 substrate but it also inhibits CYP3A4. Review of the literature about potential interactions between cyclosporine and erlotinib gave us only one published paper. De Pas et al. published that the use of cyclosporine is not a contraindication for treatment with erlotinib according to their experience and there was no relevant toxicity [9]. In their case they had to increase dose of the cyclosporine but in contrary we had to reduce it. We can assume that in our case erlotinib as a CYP34A inhibitor [7] increased the blood level of cyclosporine. Erlotinib was well tolerated in both cases and patients had only grade 1 side effects. It is important to notice that side effects have to be monitored more closely in these patients because cyclosporine is also CYP3A4 inhibitor and blood level of erlotinib can be increased because of that.

In registration studies for tyrosine kinase inhibitors median progression free survival was 9 to 11 months [10]. In our case we only have 4 months of treatment before disease progression. Zambrano Medoza et al. also published a case of early progression of EGFR positive lung adenocarcinoma in heart transplanted recipient after only 2 months of treatment with erlotinib [11]. This can be explained by advanced disease at the beginning of the treatment but there may be some other factors like immunosuppression therapy that influenced this on what we do not have any available data. In our case we did not check plasma concentration of erlotinib.

So far, we still do not know the incidence of EGFR positive patients among solid organ transplanted patients. In era of new treatment options and longer survival of solid organ transplanted patients we have to think about possible new drug interactions.

\section{Conclusions}

We can conclude that the use of erlotinib in solid organ transplanted patients treated with cyclosporine is safe and without any clinically relevant toxicity. From our experience and previously published date we recommend close monitoring of blood level of cyclosporine and erlotinib side effects.

\section{References}

1. Chapman JR, Webster AC, Wong G. Cancer in the transplant recipient. Cold Spring Harb Perspect Med 2013;3. pii: a015677.

2. Roithmaier S, Haydon AM, Loi S, et al. Incidence of malignancies in heart and/or lung transplant recipients: A singleinstitution experience. J Heart Lung Transplant 2007;26:845-9.

3. Bruschi G, Conforti S, Torre M, et al. Long-term results of lung cancer after heart transplantation: Single center 20-year experience. Lung Cancer 2009;63:146-50.

4. Crespo-Leiro MG, Villa-Arranz A, Manito-Lorite N et al. Lung cancer after heart transplantation: Results from a large Multicenter Registry. Am J Transplant 2011;11:1035-40.

5. Higgins RS, Brown RN, Chang PP, et al. A multi-institutional study of malignancies after heart transplantation and a comparison with the general United States population. J Heart Lung Transplant 2014;33:478-85.

6. Mitsudomi T. Molecular epidemiology of lung cancer and geographic variations with special reference to EGFR mutations 2014;3:205-11.

7. Peters S, Zimmermann S, Adjei AA. Oral epidermal growth factor receptor tyrosine kinase inhibitors for the treatment of non-small cell lung cancer: comparative pharmacokinetics and drug-drug interactions. Cancer Treat Rev 2014;40:917-26.

8. Miao Y, Everly JJ, Gross TG, et al. De novo cancers arising in organ transplant recipients are associated with adverse outcomes compared with the general population. Transplantation 2009;87:1347-59.

9. De Pas T, Spitaleri G, Pelosi G, et al. Erlotinib combined with cyclosporine in a liver-transplant recipient with epidermal growth factor receptor-mutated non-small cell lung cancer. J Thorac Oncol 2009;4:138-9.

10. Ellis PM, Coakley N, Feld R, et al. Use of the epidermal growth factor receptor inhibitors gefitinib, erlotinib, afatinib, dacomitinib, and icotinib in the treatment of non-small-cell lung cancer: a systematic review. Curr Oncol Tor Onc 2015;22:183-215.

11. Zambrano Mendoza E, Yen CT, Takahashi TK, et al. Metastatic lung adenocarcinoma harboring an EGFR-activating mutation in a heart transplant recipient. J Glob Oncol 2017;4:1-4. 
